\title{
Neue Fragebögen im Alltag gut anwendbar
}

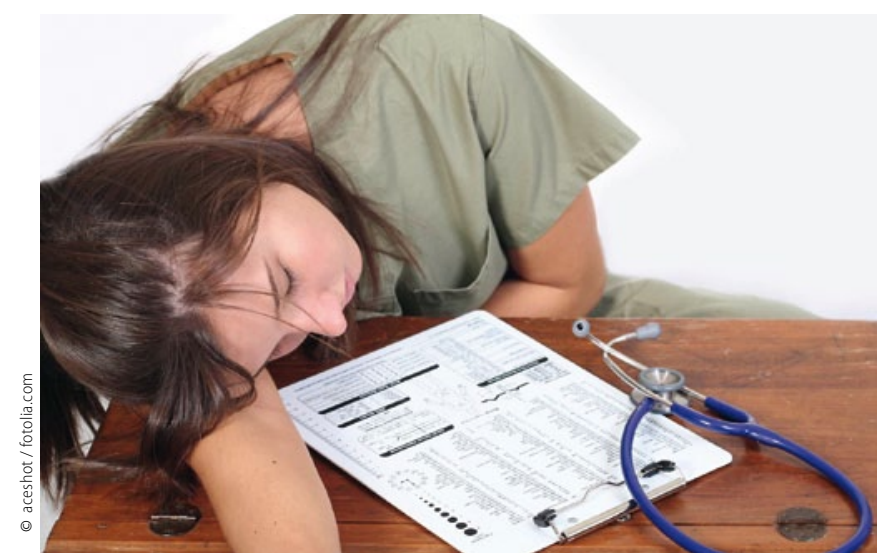

Für die neuen Screening-Scores ist es nicht mehr nötig, bis zur Erschöpfung Fragen zu beantworten.

Fragestellung: Welche nicht invasiven Hilfsmittel können ein Diabetesrisiko in der Praxis valide und sicher identifizieren?

Hintergrund: Damit Maßnahmen zur Prävention des Diabetes in der Versorgungsebene effektiv umgesetzt werden, brauchen wir einfache Hilfsmittel, um das Diabetesrisiko zu erkennen. Der Goldstandard ist der orale Glukosetoleranztest (oGTT), der als Diagnostikum für die Prävention - außer im ärztlichen Umfeld - nicht effektiv und kostengünstig anwendbar ist.

Es gibt viele Fragebögen unterschiedlichster Qualität, die anamnestische Faktoren mithilfe der Selbsteinschätzung erfragen. Verschiedene Algorithmen kalkulieren daraus ein bestehendes oder ein prospektives Diabe-

Thoopputra T, Newby D, Schneider J, Li SC. Survey of diabetes risk assessment tools: concepts, structure and performance. Diabetes Metab Res Rev 2012;28(6):485-98. tesrisiko. Häufiger Kritikpunkt ist, dass Fragebögen in einer bestimmten Population entwickelt werden. Diese können dort gut funktionieren, aber das kann in einer anderen Population vollkommen an- ders sein. So sollten Fragebögen in der jeweiligen Population, in der sie angewandt werden, auch evaluiert werden, was bisher oft nicht geschah. Die Autoren dieser Arbeit versuchten einen Überblick zu geben, um die Effektivität, aber auch Limitationen von verschiedenen Diabetesrisiko-Screening-Instrumenten darzustellen und gegebenenfalls Empfehlungen zu entwickeln, wie solche Instrumente weiterentwickelt werden können.

Methodik: Die Autoren untersuchten akribisch die wissenschaftliche Literatur aus den vergangenen 17 Jahren zu dem Thema. Es gab 2168 Artikel zur Darstellung von verschiedenen Diabetesrisiko-Screening-Tools. Diese beschäftigen sich mit 41 verschiedenen Instrumenten aus 22 Ländern, wovon der größte Teil (26) in Nordamerika und Europa entwickelt wurde.

Ergebnisse: Alle dargestellten Risikofragebögen beinhalteten 2 bis 16 Fragen. Die häufigsten Variablen waren Alter, Geschlecht, Taillenumfang, BMI, Familienanamnese für Diabetes, eigene Anamnese für Bluthochdruck oder eine Therapie mit Antihypertensiva. Die untersuchten Scores berechneten ein Diabetesrisiko auf unterschiedliche Art und Weise. Manche gaben fein abgestuft ein Diabetesrisiko an, andere nur eine einzelne Schwelle. Die Genauigkeit der Scores lag bei 40\%-97\% (Sensitivität) und $24 \%-86 \%$ (Spezifität) und der Area under the Receiver Operating Characteristic curve (ROC AUC) zwischen $62 \%$ und $87 \%$. Die Autoren stellen dar, dass das Angebot an Screening-Scores deutlich zugenommen hat und dass die Länge der Fragebögen dabei abgenommen hat.

Schlussfolgerungen: Die Autoren stellen optimistisch dar, dass die Perspektive für den Einsatz von Screening-Scores gut sei. Die neu entwickelten Scores legen zunehmend einen Fokus auf die Anwendbarkeit in der klinischen Praxis.

Dies zeigt sekundär, dass Praxiskollegen interessiert sind, ein solches Screening in der täglichen Routine anzuwenden. Die untersuchten Scores ergaben eine mittlere bis hohe Genauigkeit für die Vorhersage eines Diabetes. Häufig fehlten jedoch direkte Vergleiche zwischen den einzelnen Instrumenten.

\section{- Kommentar von Prof. Dr. med. Peter Schwarz}

\section{Scores begleitend zur Anwendung evaluieren}

Man kann davon ausgehen, dass in Zukunft weitere Scores entwickelt werden. Für die klinische Praxis ist es aber nötig, dass die Instrumente einfach transparent und gut verständlich für Arzt und Patient entwickelt werden. Vorteilhaft wäre, wenn der Einsatz begleitend evaluiert wird, sodass die Effekte prospektiv erfasst werden können. Die aktuelle Evidenz zeigt deutlich, dass Screening-Scores für das Diabetesrisiko einen substanziellen klinischen Mehrwert sowohl in der Hausarzt- als auch diabetologischen Praxis haben könnten.

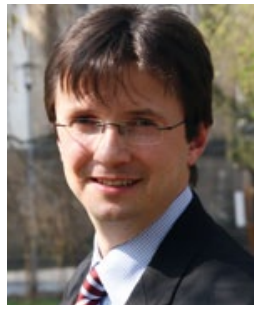

Prof. Dr. med. Peter E.H. Schwarz

Medizinische Abteilung III

Prävention und Diabetestherapie

Universität Dresden

Fetscherstr. 74

01307 Dresden

peter.schwarz@uniklinikum-dresden.de 